\title{
Thermovision and ground penetrating radar for investigation of architectural decorations - potential and limitations
}

by Adam Cupa*, Mariusz Felczak ${ }^{* * *}$, Teresa Kurkiewicz*, Angelika Lament ${ }^{*}$, Robert Rogal ${ }^{*}$, Jarosław Rogóż ${ }^{\star}$, Paweł Szroeder**, Bogusław Więcek ${ }^{\star \star *}$

* Institute for the Study, Conservation and Restoration of Cultural Property, Nicolaus Copernicus University, Torun, adamcupa@umk.pl, teresak@umk.pl, angel2312@op.pl, rr@umk.pl, jrogoz@umk.pl

** Institute of Physics, Nicolaus Copernicus University, Torun, psz@fizyka.umk.pl

*** Institute of Electronic, Technical University of Lodz, felczak@p.lodz.pl, wiecek@p.lodz.pl

\section{Abstract}

In the Institute for the Study, Conservation and Restoration of Cultural Property, Nicolaus Copernicus University, Torun, in recent years research is conducted on the application of non-destructive methods for diagnosis of historic monuments. The research presented below is the first in Poland attempt of systematic application of thermovision and radar methods for the analysis of complex structures in mural paintings.

The thermographic examination was performed on a laboratory model of architectural decoration, using thermographic cameras working in the near (NIR 0.8-1.5 $\mu \mathrm{m}$ ) and far-infrared (LWIR 7-14 $\mu \mathrm{m}$ ) and ground penetrating radar (GPR), equipped with a scanning aerial 0,9 and $2 \mathrm{GHz}$.

The model of damaged and treated polychrome plasters was created specifically for the research work and it was prepared to resemble the situations occurring in historic structures.

\section{Introduction}

Laboratory model of polychrome plaster (4m high and $3 \mathrm{~m}$ wide) was created specifically for the research work within the framework of a master diploma project. It was designed to mirror real conditions and conservation issues met in the original historic structures. The model's structure comprised different variations of plasters, polychromes with the variety of paint layers and materials employed in conservation treatment applied to imitate areas of historic mural painting that were consolidated, reattached or patched. Furthermore, there were also zones of granular disintegration, exfoliation of the paint layer and the support and also numerous cracks and damp areas. Apart of those various anomalies were included such as electric wires, screws and metal anchors, wooden slats etc. The wide diversity of model's structure was necessary for indication of crucial moments of measuring devices in order to choose optimal measurement's parameters and to work out some working procedures at the stage of interpretation of difficult conservation issues (fig. 1).

This laboratory model was exposed to thermal waves of high energy to heat up. The heating process was alternating with time what allowed for the analysis of delay of thermal response in relation to stimulation. It was established that on the basis of thermal inertia it will be possible to judge the depth and geometrical parameters of physical defects.

\section{Measuring setup}

Tests were conducted in the laboratory, where the above described model of a mural was prepared on one of the walls. The room conditions were adjusted to $39 \% \mathrm{RH}$ and $20^{\circ} \mathrm{C}$, the window was blinded to eliminate unwanted effect of solar radiation. Thermographic system used for the investigations consisted of four halogen lamps $1 \mathrm{~kW}$ each. The lamps are supplied by the driver enabling to choose a pulse duration of a thermal wave frequency. Second part of the system was an infrared camera Jenoptic VarioCam Hr. The sequences of thermographs recorded on the camera have been transferred to a computer and subject to digital analysis - a Fourier transformation - using a "Thermoscope" software.

Radar investigations were performed with the use of a GPR system made by an Italian company IDS. (fig. 2). The system consisted on a bipolar aerial emitting impulse radar waves of a frequency $2 \mathrm{GHZ}$ and a receiving aerial. The system was controlled by a computer fitted with special software responsible for acquisition and preliminary evaluation of individual radar scans. For the analysis of sequences of GPR profiles obtained from the scanned surface (the so called GPR profile networks) and for executing the 3-D maps of distribution of anomalies the GRED 3D software was employed. The GPR (ground penetrating radar) method consists on measuring the time in which the radar wave returns after being reflected on the limit of environments of different dielectric properties. This phenomenon allows for detecting the so called anomalies present below the plaster surface. 
a)

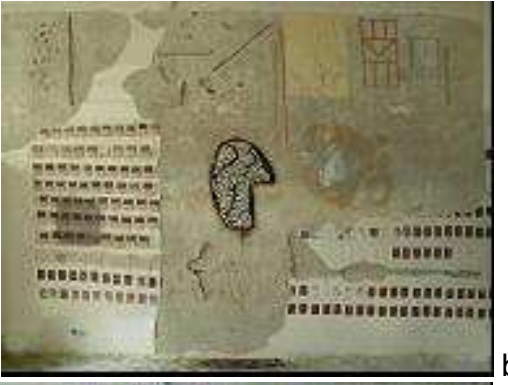

d)

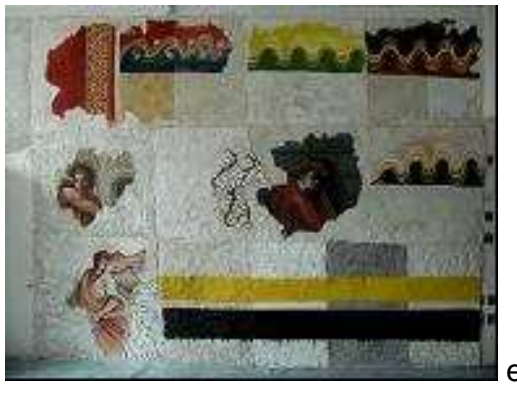

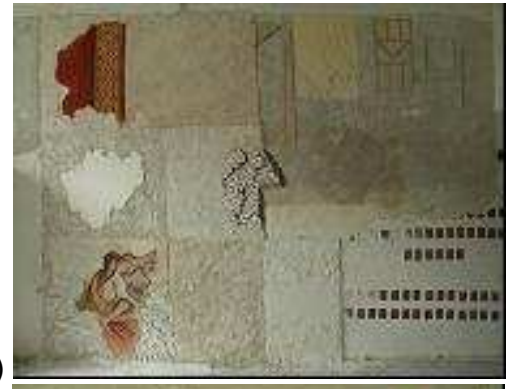
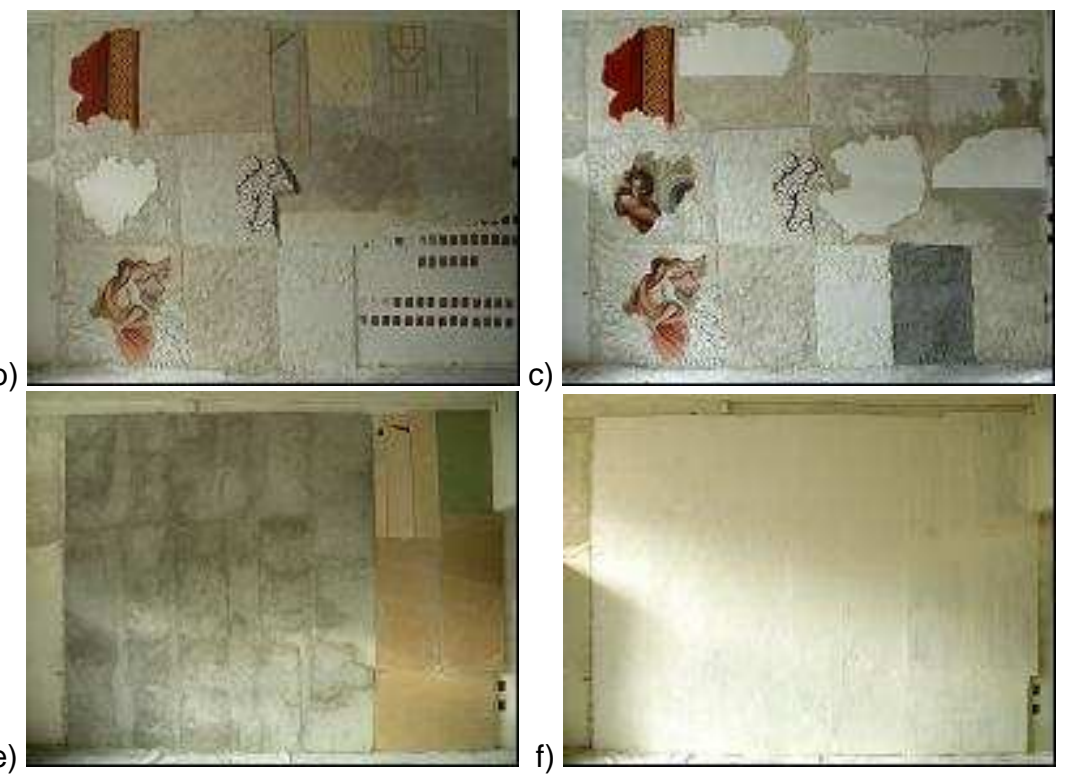

Fig. 1. Laboratory model of damaged and treated polychrome plasters - during the preparation. The photographs present subsequent stages of constructing the model - from application of first samples of paint layers, metal and wooden slats (a), trough application of lime and cement plasters as well as lime-wash and paint layers (b, c, d). Next stage consisted on applying overall layer of cement plaster and plaster-cardboard panels (e). Finally everything was white-washed (f).

\section{Examinations}

\subsection{Thermal pulse method}

Dynamic thermography is a new method of non-destructive measurements, in which an investigated object is being excited by heat energy pulses. Thermal response of the object in form of a time-variable surface temperature distribution is then recorded by thermal camera (Fig. 2). Dynamic thermography is often named pulse or lock-in thermography [11]-[15]. The example of such measurements could be the research of semiconductor and other electronic structures, where the flow of electric current is the source of thermal effects. The method can also be applied for other, non-conductive materials, for instance walls of buildings. In such case, a high power incandescent lamp is the source of energy that emits thermal radiation.

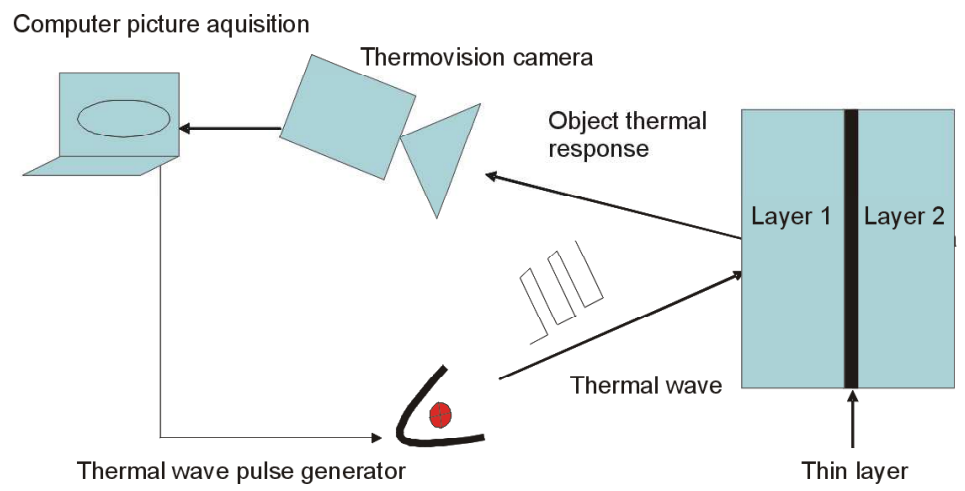

Fig. 2. Idea of non-destructive measurements thermal wave method. Exemplary computer system of pulse thermography with heat energy sources.

The heat in solid materials disperses by means of diffusion, which strongly depends on thermal parameters of the measured structures. Researchers are mainly interested in multilayer structures, in which thermal response of the front side of the object to the energy excitation depends on the parameters of the internal layers. In case of lock-in 
thermography the thermal response is periodical and synchronic with the excitation. Simple thermal analysis of the structures allows to demonstrate that not only amplitude but also thermal response phase depends on the parameters of measured materials.

There exists another type of dynamic thermography - pulse thermography, in which the excitation is a pulse of energy, a signal which includes the infinite spectrum of frequencies [12]. In this case, instead of frequency analysis, which is currently used in lock-in thermography, time constant analysis of selected elements of the structure can be used. The pulse thermography gives particularly positive results in case of measurements of thin layers.

The aim of non-destructive measurements thermal wave method is to disclose the defects in structures, define their type, size and depth, not affecting the sample. The goal of the measurement method described in this article is to find convection coefficient dependencies for a given sample on the speed of airflow around it (also for natural convection).

a)

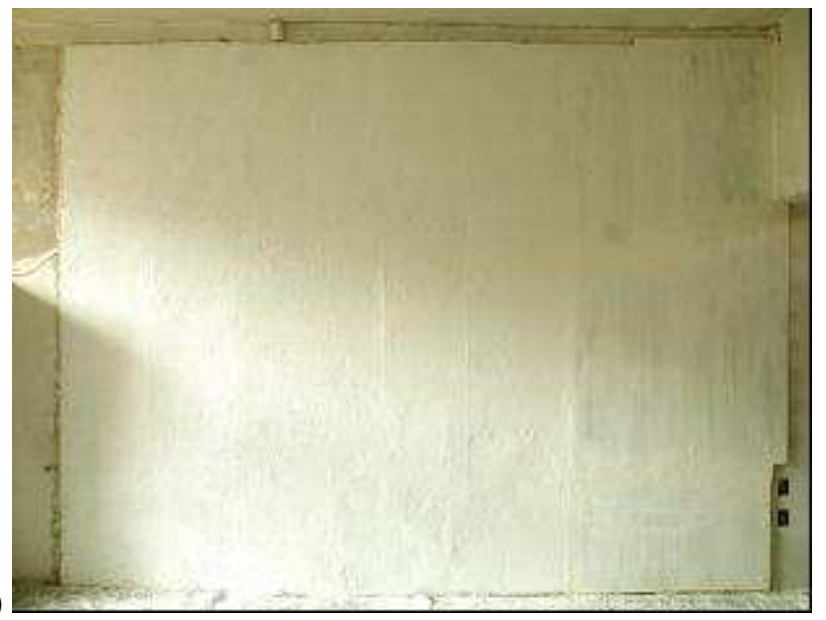

b)

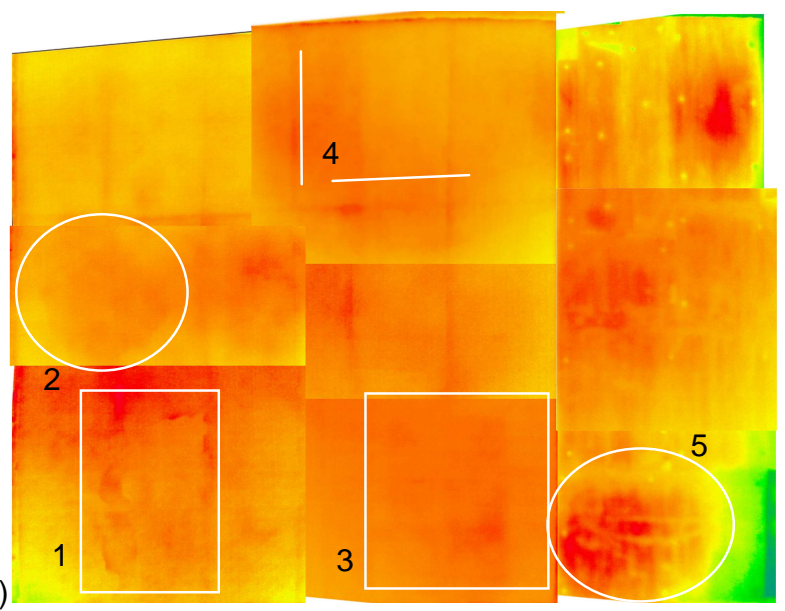

Fig. 3. Wall with the laboratory model (a), thermograph mask after thermovision scanning (b) Five anomalies, recorded with the method of synchronic thermography were marked on the thermograph.
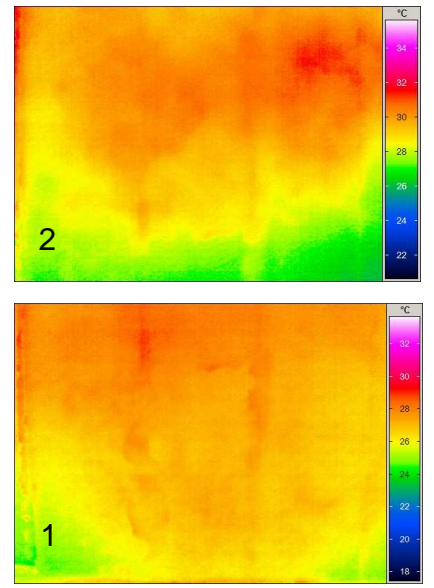

a)
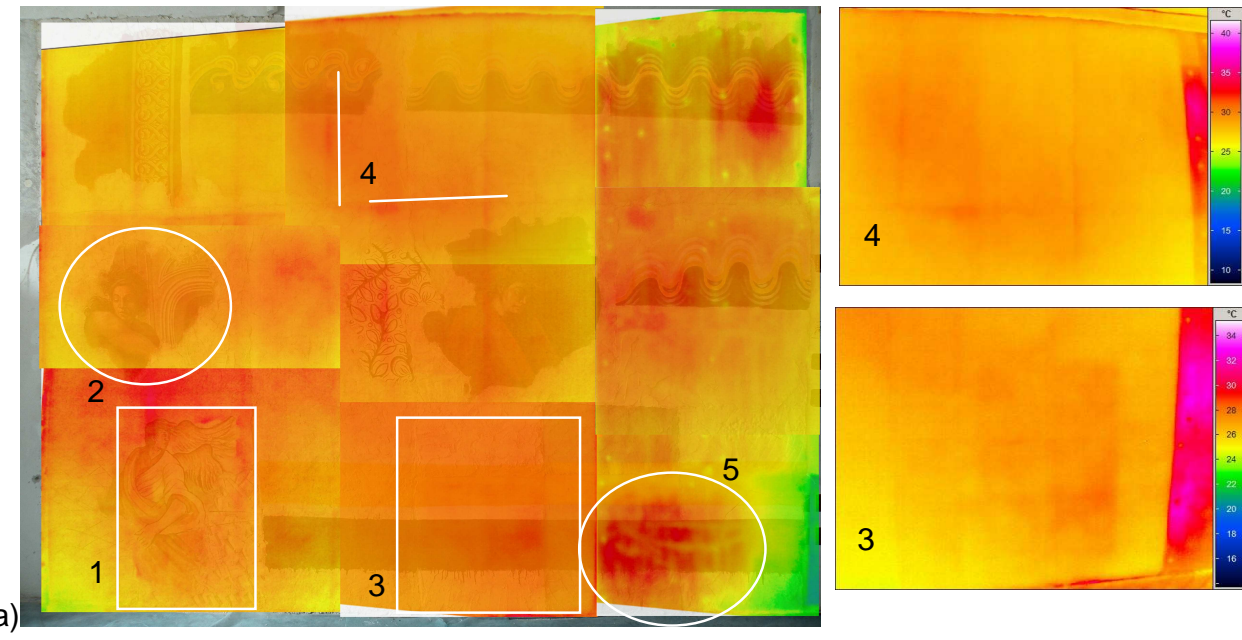

3

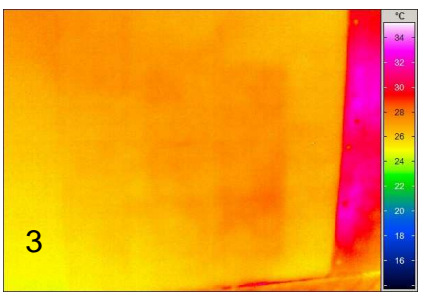

Fig. 4. Thermograph mask superimposed on the photograph of the laboratory model wall under the last layer of whitewash and finishing plaster $1,5 \mathrm{~cm}$ thick. (a.) In case of thermographs 1 and 2 the painting executed in a fresco technique, situated under the plaster and white-wash were revealed. Thermograph 3 reveals the daily limits of cement mortars (white plaster and gray - Portland - plaster), that are also situated beneath the layer of plaster. Thermograph 4 revealed regular divisions resulting from the method of applying plaster. 


\subsection{Ground Penetrating Radar method}

The GPR technique is a non-destructive research method and allows for performing analyses of the inner structure of historic objects without any mechanical damage or physical and chemical alterations of the properties of materials. The measurement consists on scanning the investigated surface with a radar aerial. The emitting aerial sends impulses of radio waves that - penetrating the investigated object - are being suppressed, multiply dispersed and reflected on the limits of two environments of different properties. The reflected waves are registered by the receiving aerial of the device.

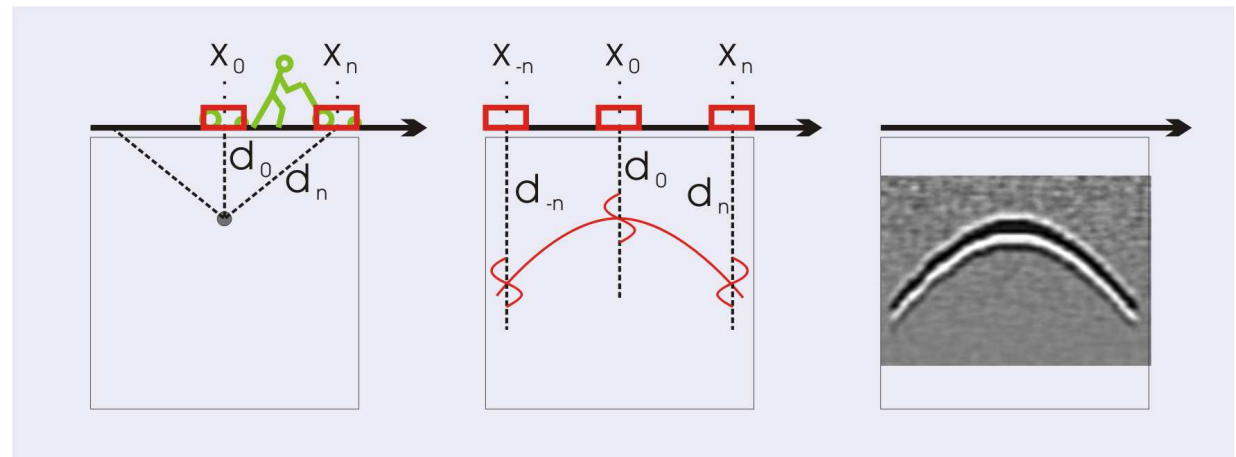

Fig. 5. Point-anomalies result in a radar image of a characteristic hyperbolic shape. With slow approach of the radar aerial towards the anomaly and with moving it away from it changes the time delay of a signal reflected by the object present in the examined structure. Signals reflected by the object create the hyperbole, the peak of which indicates the position of anomaly. The contrast of the recorder radar profile depends on the relative difference between dielectric constancies of the neighboring materials.

The image resulting from processing the signal form receiving aerial is called the radar profile. Anomalies within the examined material are represented by hyperbolic curves. The peaks of hyperboles indicate the position of anomalies below the surface. Precise localization of the revealed object in relation to reference point (initial scanning line) is possible since the aerial is fitted with measuring wheel monitoring the speed of the aerial movement. Environments examined with introspective radar are often non-homogenous. Superimposing of large number of hyperboles hinders their interpretation. That is why for the analysis of profiles the solutions borrowed from the SAR (Synthetic Aperture Radar) technique are employed.

The depth of penetration depends on the choice of an aerial of a suitable operating frequencies. By physical principles higher frequency means smaller depth of penetration giving however better resolution, that is better precision of localization and assessment of inner anomalies, defects and objects.

In the present research a bipolar aerial of $2 \mathrm{GHz}$ frequency was employed, of a $1 \mathrm{~m}$ optimum penetration range

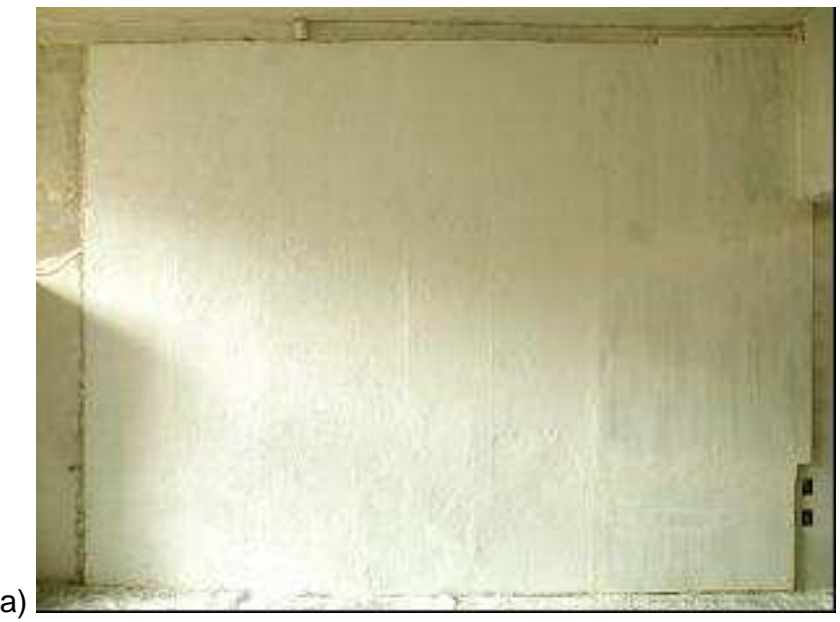

b)

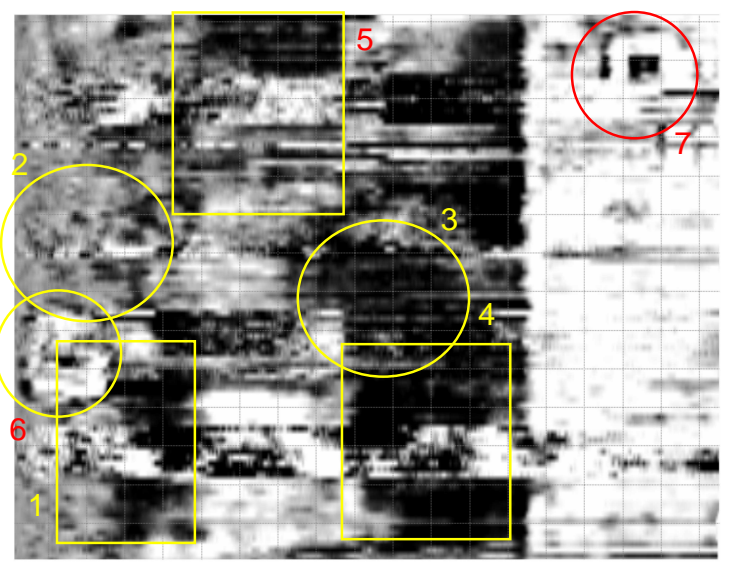

Fig. 6. Laboratory model of the wall (a), radar image from the depth of 1,58 c, (b). Five anomalies present in a subsurface structure are marked 


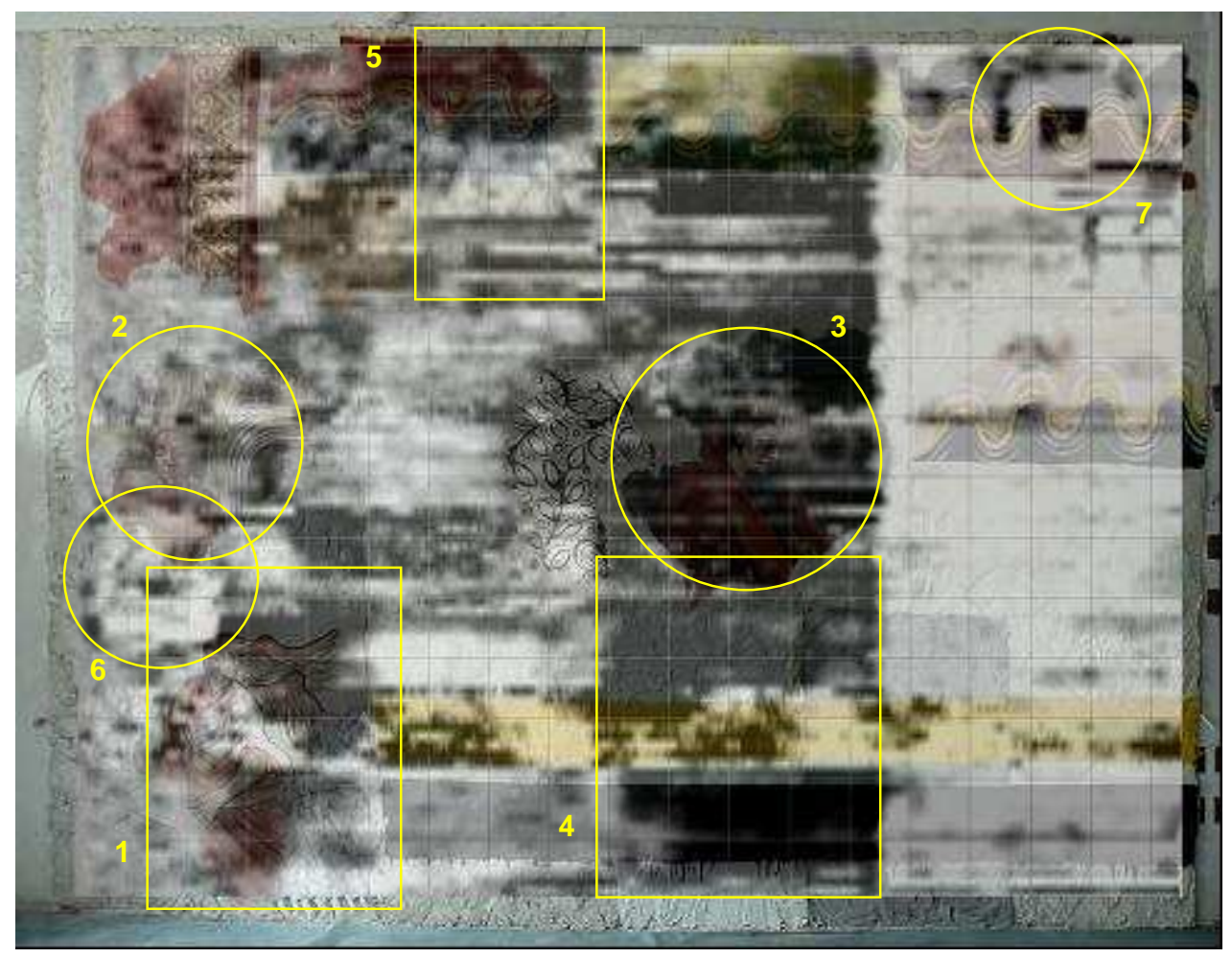

Fig. 7. Radar image from the depth of $1,58 \mathrm{~cm}$ reveals the outlines of plasters on which painting were executed (1,2, 3). In the areas 4 and 5 regular divisions are visible, resulting from the technique of applying the lower plaster layer. Metal elements present at lower depth - directly on the brick wall - give a strong echo in superficial layers (6 and 7)

\section{Conclusions}

The investigation executed on a model indicated the possibilities and limitations of both techniques. The examinations of laboratory model proved that the thermographic techniques are useful in superficial and subsurface testing up to several millimeters in the depths. However, the anomalies occurring in deeper layers may be effectively located and identified by means of GPR.

The results of an examination of the model wall allow for a conclusion, that dynamic thermographic methods allow for revealing the outlines of anomalies present in inner structure of walls, located even under $1,5 \mathrm{~cm}$ thick layers of plaster. On the other hand, the GPR technique allows for the analysis of inner structures. The analysis of processed GPR signals in form of three-dimensional maps allows for observing inner structures in sections even every 1,3 mm. This gives the possibility of precise localization and interpretation of anomalies. At this stage of research the presence of metal structures inside the examined structures is a limitation. They produce strong echo, that is visible both in layers surface plasters and on lower depth - in the wall. Such strong reflections preclude precise interpretation of the depth on which they occur. The results obtained after application of both of methods on model plaster show the direction of the research aiming to create a multimodal system, which would allow to practice an integrated thermographic and GPR examinations on the historical monuments. As it has been demonstrated it would speed up and improve the diagnostics. 


\section{REFERENCES}

[1] Avdelidis N. P., Moropoulou A., Applications of infrared thermography for the investigation of historic structures, Journal of Cultural Heritage ,vol. 5, Issue: 1, 2004, s. 119-127; Cataldo R., De Donno A., De Nunzio G., Leucci G., Nuzzo L., Siviero S., Integrated methods for analysis of deterioration of cultural heritage: the Crypt of "Cattedrale di Otranto", Journal of Cultural Heritage, vol. 6, Issue: 1, 2005, s. 29-38.

[2] [2] Cernuschi F., Ludwig N., Teruzzi P., Bottazzi G., A critical analysis and possible modifications of two analytical models for defects sizing using Video Pulse Thermography, [w:] Proceedings of Quantitative Infrared Thermography, September 7-10 1998, 1998, s. 205-209; Brink A., Maierhofer Ch., Rolling M., Wiggenhauser $\mathrm{H}$., Application of quantitative impulse thermography for structural evaluation in civil engineering - Comparison of experimental results and numerical simulations, [w:] Proceedings of Quantitative Infrared Thermography, September 24-27 2002, 2002, s. 35-40.

[3] Daniels J. J., Roberts, R. and Vendl, M., Ground Penetrating Radar for detection of liquid contaminants, Journal of Applied Geophysics, 33, 1995, 1-3, s. 195-207.

[4] Daniels D. J., Ground Penetrating Radar - 2nd Edition, The Institution of Electrical Engineers, London 2004.

[5] Rogoz J. The application of non-destructive techniques in conservation research of murals, Wydawnictwo Naukowe UMK, Toruń 2009.

[6] Bartz W., Rogóż J., Rogal R., Cupa A., Szroeder P., Characterization of historical lime plasters by combined non-destructive and destructive tests: The case of the sgraffito in Bożnów (SW Poland), Construction and Building Materials, 30, 2012, Elsevier, s. 439-446.

[7] Rogal R., Rogóż J., Szroeder P., Bartz W., Cupa A., Application of novel nondestructive techniques to investigations of historical plasters: a case study of sgraffito decoration in Bożnów, Measurement Automation and Monitoring, nr 8, 2010, s. $961-964$.

[8] B. Więcek, T. Wajman, G. Gralewicz, S. Zwolenik; „Theory and measurements of single and multilayer structures using lock-in and pulse thermography"; Thermosense XXV Conference, 21-25 April 2003, Orlando, Florida, USA.

[9] B. Więcek, T. Wajman, G. Gralewicz, M. Michalak; „Multilayer structure investigations using lock-in and pulse thermography - possible applications in medicine"; $25^{\text {th }}$ Annual Internatinal Conference of the IEEE Engineering in Medicine and Biology Society, 17 - 21-09-2003, Cancun, Mexico

[10] B. Więcek, T. Wajman, G. Gralewicz; „Application of Thermal Wave Methods to Electronics”; MICROTHERM Conference "Thermal problems in electronics", 29.06 - 2.07. 2003, Łódź.

[11] D. P. Almond, and P. M. Patel - Photothermal science and techniques. London, chapman \& Hall, 1996, p. 108.

[12] V. Vavilov, S. Marinetti, E. Grinzato And P. Bison - Thermal tomography, characterisation and pulse phase thermography of impact damage in CFRP, or why end-users are still reluctant about practical use of transient IR thermography. Snell (J.R.) and Wurzbach (R.N.) eds., Thermosense-XX, SPIE Proc. 3361, 1998, p.275-281.

[13] J. Rantala, D. Wu And G. Busse - NDT of polymer materials using lock-in thermography with water-coupled ultrasonic excitation. NDE \& E. Intern., 31, 1998, No.1, p.43-49.

[14] D. P. Almond, and C. K. Lau - Defect sizing by transient thermography. I. An analytical treatment. J. Phys. D: Appl. Phys., 27, 1994, p.1063-1069.

[15] D. Wu, A. Salerno, B. Schönbach, H. Halin And G. Busse - Phase-sensitive modulation thermography and its applications for NDE. Wurzbach (R.N.) 